\title{
SOME IRREDUCIBLE REPRESENTATIONS OF BRAUER'S CENTRALIZER ALGEBRAS
}

\author{
JUN HU \\ Department of Mathematics, Beijing Normal University, Beijing, 100875, P. R. China \\ e-mail: junhu303@yahoo.com.cn \\ and YICHUAN YANG \\ Institut für Algebra und Zahlentheorie, Fachbereich Mathematik, Universität Stuttgart, \\ Pfaffenwaldring 57, Stuttgart, 70569, Germany \\ e-mail: yyc@mathematik.uni-stuttgart.de
}

(Received 3 August, 2003; accepted 3 March, 2004)

\begin{abstract}
Let $m, n \in \mathbb{N}, V$ be a $2 m$-dimensional complex vector space. The irreducible representations of the Brauer's centralizer algebra $B_{n}(-2 m)$ appearing in $V^{\otimes n}$ are in $1-1$ correspondence to the set of pairs $(f, \lambda)$, where $f \in \mathbb{Z}$ with $0 \leq f \leq[n / 2]$, and $\lambda \vdash n-2 f$ satisfying $\lambda_{1} \leq m$. In this paper, we first show that each of these representations has a basis consists of eigenvectors for the subalgebra of $B_{n}(-2 m)$ generated by all the Jucys-Murphy operators, and we determine the corresponding eigenvalues. Then we identify these representations with the irreducible representations constructed from a cellular basis of $B_{n}(-2 m)$. Finally, an explicit description of the action of each generator of $B_{n}(-2 m)$ on such a basis is also given, which generalizes earlier work of [15] for Brauer's centralizer algebra $B_{n}(m)$.
\end{abstract}

2000 Mathematics Subject Classification. 16G99.

1. Introduction. Let $m, n \in \mathbb{N}, V$ be a $m$-dimensional (resp. $2 m$-dimensional) complex vector space with a non-degenerate symmetric (resp. skew) bilinear form. Then $V$ defines the orthogonal group $O(V)$ (resp. symplectic group $S p(V)$ ), the invertible linear transformations which preserve this form.

In order to study how the $n$-tensor space $V^{\otimes n}$ decompose into irreducible modules over $O(V)$ or $S p(V)$, Richard Brauer (see [1]) introduced a number of complex associative algebras $B_{n}(x)$ which are now called the Brauer's centralizer algebras. These algebras are finite-dimensional algebras indexed by a positive integer $n$ and a complex number $x$.

One takes $x=m$ in the orthogonal case, and $x=-2 m$ in the symplectic case. Then there is a right action of $B_{n}(x)$ on the corresponding $n$-tensor space $V^{\otimes n}$, such that it generates the centralizer algebra $\operatorname{End}_{O(V)}\left(V^{\otimes n}\right) \operatorname{or}_{\operatorname{End}_{S p(V)}}\left(V^{\otimes n}\right)$.

Research supported by Alexander von Humboldt Foundation. The second author acknowledges support from DAAD. Both authors wish to thank the referee for helpful comments, allowing them to clarify their treatment in the first version of this paper. 
In this paper, we shall only be concerned with the symplectic case ${ }^{1}$.

TheOREM $1.1\left(\right.$ (11], [2], [3]). Let $\varphi: B_{n}(-2 m) \rightarrow \operatorname{End}_{S p(V)}\left(V^{\otimes n}\right)$ and $\psi: \mathbb{C S p}(V) \rightarrow$ $\operatorname{End}_{B_{n}(-2 m)}\left(V^{\otimes n}\right)$ be the natural algebra homomorphisms.

(1) Both $\varphi$ and $\psi$ are surjective, and if $m \geq n$ then $\varphi$ is an isomorphism.

(2) There is an irreducible $\left(\mathbb{C} S p(V),\left(B_{n}(-2 m)\right)^{\text {op }}\right)$-bimodules decomposition

$$
V^{\otimes n}=\bigoplus_{\substack { f=0 \\
\begin{subarray}{c}{\lambda \vdash n-2 f \\
\lambda_{1} \leq m{ f = 0 \\
\begin{subarray} { c } { \lambda \vdash n - 2 f \\
\lambda _ { 1 } \leq m } }\end{subarray}}^{[n / 2]} U^{\lambda} \otimes V^{\lambda},
$$

where $U^{\lambda}\left(\right.$ resp. $\left.V^{\lambda}\right)$ is an irreducible $\operatorname{Sp}(V)$-module (resp. an irreducible $B_{n}(-2 m)$ module) corresponding to $\lambda$.

Note that, unlike the symmetric group algebra, the Brauer's centralizer algebra $B_{n}(-2 m)$ over $\mathbb{C}$ in the above theorem is not necessarily semisimple. But its image under $\varphi$, the centralizer algebra $\operatorname{End}_{S p(V)}\left(V^{\otimes n}\right)$, is always semisimple. It is for this reason that the $n$-tensor space $V^{\otimes n}$ is completely reducible as a module over the Brauer's centralizer algebras $B_{n}(-2 m)$.

The dimensions of these irreducible $B_{n}(-2 m)$-modules $V^{\lambda}$ are already known. See (3.4) and [18] for the definition of permissible up-down tableaux.

THEOREM $1.2([\mathbf{1 8}])$. For any integer $f$ with $0 \leq f \leq[n / 2]$ and any partition $\lambda$ of $n-2 f$ satisfying $\lambda_{1} \leq m$, we have that

$$
\operatorname{dim} V^{\lambda}=\#\{(-2 m) \text {-permissible up-down tableaux of shape } \lambda \text { and length } n .\}
$$

In [15], M. Nazarov introduced a remarkable family of pairwise commuting elements in the Brauer's centralizer algebra, which are called Jucys-Murphy operators. Using Schur-Weyl duality between orthogonal groups $O_{m}(\mathbb{C})$ and Brauer's centralizer algebras $B_{n}(m)$, he showed that each irreducible representation of $B_{n}(m)$ appearing in $V^{\otimes n}$ has a canonical basis (whose vectors are defined up to scalar multipliers) consisting of common eigenvectors for all the Jucys-Murphy operators, and he gave an explicit description of the action of each generator of $B_{n}(m)$ on such a basis. Note that for $m \geq n$, this description is already known by [14] (see also [11]). But Nazarov's results are valid for any integer $m$.

It is natural to ask for a similar result for the algebra $B_{n}(-2 m)$, which is one starting point of this work. On the other hand, as it is well-known (see [7]), the Brauer's centralizer algebra $B_{n}(x)$ (for any parameter $x$ ) is always a cellular algebra. Hence the general theory of cellular algebra can apply. One has cell modules, and modular irreducible modules which are defined to be some quotients of cell modules modulo the radicals of certain naturally defined bilinear forms. Therefore one can also ask what those irreducible representations appearing in $V^{\otimes n}$ should look like in this picture. Using Jucys-Murphy operators in the Brauer's centralizer algebra $B_{n}(-2 m)$ and Schur-Weyl duality between the symplectic group $S p_{2 m}(\mathbb{C})$ and $B_{n}(-2 m)$, we will answer these two questions in this paper.

The content is organized as follows: in Section two we recall the definition of Brauer's centralizer algebra and the action of $B_{n}(-2 m)$ on $n$-tensor space $V^{\otimes n}$.

${ }^{1}$ The results for the orthogonal case are similar; see [1], [2] and [3]. 
In Section three we first prove that the action of the sum of all the Jucys-Murphy operators on $V^{\otimes n}$ coincides up to sign with the action of a Casimir element in the universal enveloping algebra $U\left(s p_{2 m}\right)$ for the symplectic Lie algebra. Then we show that each irreducible representation of $B_{n}(-2 m)$ found in $V^{\otimes n}$ has a canonical basis (whose vectors are defined up to scalar multipliers) consisting of common eigenvectors of all the Jucys-Murphy operators. In Section four we identify these representations with the irreducible representations constructed from a cellular basis of $B_{n}(-2 m)$. In the final section, we shall, following the approach of [15], give an explicit description of the action of each generator of $B_{n}(-2 m)$ on the canonical basis of each irreducible representations appeared in $V^{\otimes n}$.

2. Brauer's centralizer algebra. Let $x$ be an indeterminate over $\mathbb{Z}$. The (generic) Brauer's centralizer algebra $B_{n}(x)$ can be defined as a $\mathbb{Z}[x]$-algebra with generators $s_{1}, \ldots, s_{n-1}, e_{1}, \ldots, e_{n-1}$ and relations:

$$
\begin{array}{cl}
s_{i}^{2}=1, e_{i}^{2}=x e_{i}, e_{i} s_{i}=e_{i}=s_{i} e_{i}, & \forall 1 \leq i \leq n-1, \\
s_{i} s_{j}=s_{j} s_{i}, s_{i} e_{j}=e_{j} s_{i}, e_{i} e_{j}=e_{j} e_{i}, & \forall 1 \leq i<j-1 \leq n-2, \\
s_{i} s_{i+1} s_{i}=s_{i+1} s_{i} s_{i+1}, e_{i} e_{i+1} e_{i}=e_{i}, e_{i+1} e_{i} e_{i+1}=e_{i+1}, & \forall 1 \leq i \leq n-2, \\
s_{i} e_{i+1} e_{i}=s_{i+1} e_{i}, e_{i+1} e_{i} s_{i+1}=e_{i+1} s_{i}, & \forall 1 \leq i \leq n-2 .
\end{array}
$$

One can also define $B_{n}(x)$ in terms of Brauer $n$-diagrams and its multiplication in terms of concatenation of Brauer $n$-diagrams. We omit the details (as it is wellknown) and refer the readers to [1] and [8].

The algebra $B_{n}(x)$ is a free $\mathbb{Z}[x]$-module of rank $(2 n-1)(2 n-3) \cdots 3 \cdot 1$, and the subalgebra generated by $s_{1}, \ldots, s_{n-1}$ is isomorphic to the group algebra of the symmetric group $\mathfrak{S}_{n}$. For any noetherian commutative domain $R$ which is a $\mathbb{Z}[x]$-algebra, we define $B_{n}(x)_{R}=B_{n}(x) \otimes_{\mathbb{Z}[x]} R$. It is also wellknown that $B_{n}(x)_{R}$ is canonically isomorphic to the algebra (over $R$ ) presented by the same generators and relations as above.

In this paper, we will only be concerned with the Brauer's centralizer algebra $B_{n}(x)$ over the complex numbers field $\mathbb{C}$. Then $\mathbb{C}$ is a $\mathbb{Z}[x]$-algebra and we have an evaluation homomorphism $\mathbb{Z}[x] \rightarrow \mathbb{C}$. By abuse of notation, we denote again by $x$ its image in $\mathbb{C}$. It is wellknown that ([18]) $B_{n}(x)$ is semisimple if $x$ is not an integer. So we will only be interested in the case where $x$ is an integer in $\mathbb{C}$. For certain integers $x$, there are SchurWeyl dualities between orthogonal groups (or symplectic groups) and corresponding Brauer's centralizer algebras $B_{n}(x)$.

In the orthogonal case one takes $x$ to be a positive integer, which was studied in [15]. In this paper we will only be concerned with the symplectic case, i.e., $x$ is a negative even integer. Let $m \in \mathbb{N}, V$ be $2 m$-dimensional complex vector space with a nondegenerate skew bilinear form (, ). Then the symplectic group relative to $($,$) is$ defined to be $\operatorname{Sp}(V):=\{g \in G L(V) \mid(g v, g w)=(v, w), \forall v, w \in V\}$.

We fixed an ordered basis $\left\{v_{i}\right\}_{i=1}^{2 m}$ of $V$ such that

$$
\left(v_{i}, v_{j}\right)=0=\left(v_{2 m+1-i}, v_{2 m+1-j}\right), \quad\left(v_{i}, v_{2 m+1-j}\right)=\delta_{i j}, \quad \forall 1 \leq i, j \leq m .
$$

For simplicity, we shall write $i^{\prime}:=2 m+1-i$ for each $1 \leq i \leq 2 m$. There is a right action of the Brauer's centralizer algebra $B_{n}(-2 m)$ on $n$-tensor spaces $V^{\otimes n}$. The definition depends on the above choice of orthogonal basis with respect to (, ). Let $\delta_{i j}$ 
denote the value of the usual Kronecker function. Let $I:=\left\{1, \ldots, m, m^{\prime}, \ldots, 1^{\prime}\right\}$. For any $i, j \in I$, let

$$
\epsilon_{i j}:= \begin{cases}1 & \text { if } j=i^{\prime} \\ -1 & \text { if } i=j^{\prime} \\ 0 & \text { otherwise }\end{cases}
$$

The right action of $B_{n}(-2 m)$ on $V^{\otimes n}$ is defined on generators by

$$
\begin{gathered}
\left(v_{i_{1}} \otimes \cdots \otimes v_{i_{n}}\right) s_{j}:=-\left(v_{i_{1}} \otimes \cdots \otimes v_{i_{j-1}} \otimes v_{i_{j+1}} \otimes v_{i_{j}} \otimes v_{i_{j+2}} \otimes \cdots \otimes v_{i_{n}}\right) \\
\left(v_{i_{1}} \otimes \cdots \otimes v_{i_{n}}\right) e_{j}:=\epsilon_{i_{j} i_{j+1}} v_{i_{1}} \otimes \cdots \otimes v_{i_{j-1}} \otimes\left(\sum_{k=1}^{m}\left(v_{k^{\prime}} \otimes v_{k}-v_{k} \otimes v_{k^{\prime}}\right)\right) \otimes v_{i_{j+2}} \\
\otimes \cdots \otimes v_{i_{n}} .
\end{gathered}
$$

Then this right action of $B_{n}(-2 m)$ commutes with the natural left action of $\operatorname{Sp}(V)$ on $V^{\otimes n}$.

3. Jucys-Murphy operators and Casimir element. Recall that we have fixed an ordered basis $\left\{v_{1}, \ldots, v_{m}, v_{m^{\prime}}, \ldots, v_{1^{\prime}}\right\}$ of $V$. The bilinear form $($,$) relative to this basis$ is given by the block matrix

$$
J:=\left(\begin{array}{cc}
0 & J_{m} \\
-J_{m} & 0
\end{array}\right)
$$

where $J_{m}$ is the unique antidiagonal $m \times m$ permutation matrix.

Let $\epsilon_{1}, \ldots, \epsilon_{2 m}$ be the standard basis of $\mathbb{C}^{2 m}$. We identify $V$ with $\mathbb{C}^{2 m}$ (and hence $G L(V)$ with $G L_{2 m}(\mathbb{C})$ ) by mapping $v_{i}$ to $\epsilon_{i}$ for each $1 \leq i \leq 2 m$. Then $S p(V)$ is identified with $\operatorname{Sp}_{2 m}(\mathbb{C}):=\left\{A \in G L_{2 m}(\mathbb{C}) \mid A^{T} J A=J\right\}$. The symplectic Lie algebra $s p_{2 m}(\mathbb{C})$ is defined as $s p_{2 m}(\mathbb{C}):=\left\{A \in M_{2 m}(\mathbb{C}) \mid A^{T} J+J A=0\right\}$.

For each $1 \leq i, j \leq 2 m$, let $E_{i, j}$ be the $2 m \times 2 m$ matrix with an 1 in the $(i, j)$ position as its unique nonzero entry. It is easy to see that $s p_{2 m}(\mathbb{C})$ is a finite dimensional $\mathbb{C}$-vector space with basis

$$
\begin{cases}\frac{E_{i, j}-E_{j^{\prime}, i^{\prime}}}{\sqrt{2}}, & 1 \leq i, j \leq m, \\ \frac{E_{i, j^{\prime}}+E_{j, i^{\prime}}}{\sqrt{2}}, & 1 \leq i<j \leq m, \\ \frac{E_{i^{\prime}, j}+E_{j^{\prime}, i}}{\sqrt{2}}, & 1 \leq i<j \leq m, \\ E_{i, i^{\prime}}, \quad E_{i^{\prime}, i}, & 1 \leq i \leq m .\end{cases}
$$

Let $U\left(s p_{2 m}\right)$ be the universal enveloping algebra of $s p_{2 m}(\mathbb{C})$. By differentiating, one gets a natural action of $s p_{2 m}(\mathbb{C})$ (and hence of $U\left(s p_{2 m}\right)$ ) on $V^{\otimes n}$. The Casimir element of 
$U\left(s p_{2 m}\right)$ is defined as

$$
\begin{aligned}
C:= & \frac{1}{2} \sum_{1 \leq i, j \leq m}\left(E_{i, j}-E_{j^{\prime}, i^{\prime}}\right)\left(E_{j, i}-E_{i^{\prime}, j^{\prime}}\right)+\frac{1}{2} \sum_{1 \leq i<j \leq m}\left(E_{i, j^{\prime}}+E_{j, i^{\prime}}\right)\left(E_{i^{\prime}, j}+E_{j^{\prime}, i}\right) \\
& +\frac{1}{2} \sum_{1 \leq i<j \leq m}\left(E_{i^{\prime}, j}+E_{j^{\prime}, i}\right)\left(E_{i, j^{\prime}}+E_{j, i^{\prime}}\right)+\sum_{1 \leq i \leq m}\left(E_{i, i^{\prime}} E_{i^{\prime}, i}+E_{i^{\prime}, i} E_{i, i^{\prime}}\right) .
\end{aligned}
$$

Following Nazarov [15], we define

$$
\begin{aligned}
L_{1}= & \frac{-2 m-1}{2}, \\
L_{a}:= & \frac{-2 m-1}{2}+s_{a-1}+s_{a-2} s_{a-1} s_{a-2}+\cdots+s_{1} s_{2} \cdots s_{a-2} s_{a-1} s_{a-2} \cdots s_{2} s_{1} \\
& -\left(e_{a-1}+s_{a-2} e_{a-1} s_{a-2}+\cdots+s_{1} s_{2} \cdots s_{a-2} e_{a-1} s_{a-2} \cdots s_{2} s_{1}\right),
\end{aligned}
$$

for any integer $a$ with $2 \leq a \leq n$. We call the elements $L_{1}, L_{2}, \ldots, L_{n}$ the Jucys-Murphy operators for the Brauer's centralizer algebra $B_{n}(-2 m)$. These elements behave very much like the Jucys-Murphy operators for symmetric groups and associated IwahoriHecke algebras (see [12]). For example, it is shown (in [15]) that $L_{1}, L_{2}, \ldots, L_{n}$ pairwise commute with each other and the elements $L_{1}^{i}+\cdots+L_{n}^{i}$ with $i=1,3, \ldots$ are central in $B_{n}(-2 m)$. We have the following result ${ }^{2}$.

THEOREM 3.1. For any integers $i_{1}, \ldots, i_{n} \in\{1,2, \ldots, 2 m\}$, we have that

$$
\left(v_{i_{1}} \otimes \cdots \otimes v_{i_{n}}\right)\left(L_{1}+\cdots+L_{n}\right)=-C\left(v_{i_{1}} \otimes \cdots \otimes v_{i_{n}}\right) .
$$

Proof. We use induction on $n$. If $n=1$, then

$$
\begin{gathered}
v_{i_{1}} L_{1}=v_{i_{1}} \frac{-2 m-1}{2}=-\left(m+\frac{1}{2}\right) v_{i_{1}}, \\
-C\left(v_{i_{1}}\right)=-\left(\frac{m}{2}+\frac{m-i_{1}+i_{1}-1}{2}+1\right)=-\left(m+\frac{1}{2}\right) v_{i_{1}} .
\end{gathered}
$$

So the theorem is true in this case. Suppose that the theorem is true for $n-1$. That is, $\left(v_{i_{1}} \otimes \cdots \otimes v_{i_{n-1}}\right)\left(L_{1}+\cdots+L_{n-1}\right)=-C\left(v_{i_{1}} \otimes \cdots \otimes v_{i_{n-1}}\right)$, for any integers $i_{1}, \ldots, i_{n-1} \in\{1,2, \ldots, 2 m\}$.

Now for any integers $i_{1}, \ldots, i_{n} \in\{1,2, \ldots, 2 m\}$, we have that

$$
\begin{aligned}
& \left(v_{i_{1}} \otimes \cdots \otimes v_{i_{n}}\right)\left(L_{1}+\cdots+L_{n}\right) \\
& \quad=\left(v_{i_{1}} \otimes \cdots \otimes v_{i_{n}}\right)\left(L_{1}+\cdots+L_{n-1}\right)+\left(v_{i_{1}} \otimes \cdots \otimes v_{i_{n}}\right) L_{n} \\
& \quad=\left(\left(v_{i_{1}} \otimes \cdots \otimes v_{i_{n-1}}\right)\left(L_{1}+\cdots+L_{n-1}\right)\right) \otimes v_{i_{n}}+\left(v_{i_{1}} \otimes \cdots \otimes v_{i_{n}}\right) L_{n} \\
& \quad=-C\left(v_{i_{1}} \otimes \cdots \otimes v_{i_{n-1}}\right) \otimes v_{i_{n}}+\left(v_{i_{1}} \otimes \cdots \otimes v_{i_{n}}\right) L_{n} .
\end{aligned}
$$

\footnotetext{
${ }^{2}$ In the orthogonal case (see the proof $[15,(2.6)]$ ), the action of the sum of all the Jucys-Murphy operators coincides exactly (i.e., without a sign) with the action of a Casimir element $C$ of the universal enveloping algebra $U\left(s o_{m}\right)$ on $V^{\otimes n}$.
} 
Now by a direct calculation, one can show that

$$
C\left(v_{i_{1}} \otimes \cdots \otimes v_{i_{n}}\right)-C\left(v_{i_{1}} \otimes \cdots \otimes v_{i_{n-1}}\right) \otimes v_{i_{n}}=-\left(v_{i_{1}} \otimes \cdots \otimes v_{i_{n}}\right) L_{n},
$$

as required. This completes the proof of the theorem.

COROLlaRY 3.3. Let $\lambda$ be a partition of $n-2 f$ (where $f$ is an integer with $0 \leq f \leq$ $[n / 2])$ satisfying $\lambda_{1} \leq m$. Then the central element $L_{1}+\cdots+L_{n}$ acts as the scalar

$$
\left(-\frac{1}{2}-m\right)(n-2 f)+\sum_{(i, j) \in[\lambda]}(j-i)
$$

on $V^{\lambda}$.

Proof. Let $V^{\lambda}(n-2 f)$ be the irreducible representation of $B_{n-2 f}(-2 m)$ corresponding to $\lambda$ and occurring in $V^{\otimes n-2 f}$. Let $V^{\lambda}:=V^{\lambda}(n)$ be the irreducible representation of $B_{n}(-2 m)$ corresponding to $\lambda$ and occurring in $V^{\otimes n}$. Let $w$ be any nonzero element in $V^{\lambda}$. By [17], any vector in $U^{\lambda} \otimes V^{\lambda}(n-2 f)$ is traceless (w.r.t. $\left.B_{n-2 f}(-2 m)\right)$. That is, $\left(U^{\lambda} \otimes V^{\lambda}(n-2 f)\right) e_{i}=0$ for any integer $i$ with $1 \leq i<n-2 f$. Therefore, for any $0 \neq v \in U^{\lambda}, 0 \neq w^{\prime} \in V^{\lambda}(n-2 f)$, we have (by Theorem 3.1)

$$
\begin{aligned}
-C\left(v \otimes w^{\prime}\right) & =\left(v \otimes w^{\prime}\right)\left(L_{1}+\cdots+L_{n-2 f}\right) \\
& =\left(v \otimes w^{\prime}\right)\left(\frac{-2 m-1}{2}(n-2 f)+\widetilde{L}_{2}+\cdots+\widetilde{L}_{n-2 f}\right) \\
& =\left(v \otimes w^{\prime}\right)\left(\frac{-2 m-1}{2}(n-2 f)+\sum_{(i, j) \in[\lambda]}(j-i)\right),
\end{aligned}
$$

where $\widetilde{L}_{a}:=s_{a-1}+s_{a-2} s_{a-1} s_{a-2}+\cdots+s_{1} s_{2} \cdots s_{a-2} s_{a-1} s_{a-2} \cdots s_{2} s_{1}$ (for each integer $a$ with $2 \leq a \leq n-2 f$ ) is the usual Jucys-Murphy operator of the symmetric group algebra $\mathbb{C S}_{n-2 f}$. Note that the Casimir element $C$ is in the center of $U\left(s p_{2 m}\right)$ and hence acts as a scalar on irreducible module $U^{\lambda}$. It follows that

$$
-C v=\left(\frac{-2 m-1}{2}(n-2 f)+\sum_{(i, j) \in[\lambda]}(j-i)\right) v .
$$

Now applying Theorem 3.1 again, one gets that

$$
\begin{aligned}
(v \otimes w)\left(L_{1}+\cdots+L_{n}\right) & =-C(v \otimes w)=-C v \otimes w \\
& =\left(\frac{-2 m-1}{2}(n-2 f)+\sum_{(i, j) \in[\lambda]}(j-i)\right)(v \otimes w) .
\end{aligned}
$$

In particular, we get that

$$
w\left(L_{1}+\cdots+L_{n}\right)=\left(\left(-\frac{1}{2}-m\right)(n-2 f)+\sum_{(i, j) \in[\lambda]}(j-i)\right) w
$$

as required. 
DEFINITION 3.4 ([18]). Let $\lambda$ be a partition of $n-2 f$ (where $f$ is an integer with $0 \leq f \leq[n / 2])$ satisfying $\lambda_{1} \leq m$. A $(-2 m)$-permissible up-down tableau of shape $\lambda$ and length $n$ is a sequence $\Lambda=(\emptyset=\Lambda(0), \Lambda(1), \ldots, \Lambda(n)=\lambda)$ of partitions, such that for each $1 \leq k \leq n, \Lambda(k)_{1} \leq m$ and the Young diagram $[\Lambda(k)]$ differs from the Young diagram $[\Lambda(k-1)]$ by either adding or removing a box. We denote by $\operatorname{UD}_{n, \lambda}(-2 m)$ the set of all the $(-2 m)$-permissible up-down tableaux of shape $\lambda$ and length $n$.

Definition 3.5. Let $\Lambda=(\emptyset=\Lambda(0), \Lambda(1), \ldots, \Lambda(n))$ be a given $(-2 m)$ permissible up-down tableau. Let $a$ be any integer with $1 \leq a \leq n$. Suppose that $\Lambda(a-1)$ differs from $\Lambda(a)$ by a box in row $i$ and column $j$. Then the residue of $a$ in $\Lambda$ is defined to be $(-1 / 2)-m+j-i$ if $\Lambda(a) \supset \Lambda(a-1)$; or $(1 / 2)+m+i-j$ if $\Lambda(a) \subset \Lambda(a-1)$. We denote it by $\operatorname{res}_{\Lambda}(a)$.

Let $f$ be an integer with $0 \leq f \leq[n / 2]$. For each partition $\lambda$ of $n-2 f$ satisfying $\lambda_{1} \leq m$, we have that (by [18]) $\operatorname{dim} V^{\lambda}=\# \operatorname{UD}_{n, \lambda}(-2 m)$.

THEOREM 3.6. For any partition $\lambda$ of $n-2 f$ (where $f$ is an integer with $0 \leq f \leq[n / 2]$ ) satisfying $\lambda_{1} \leq m$, there is a canonical basis (whose vectors are defined up to scalar multipliers) of $V^{\lambda}$, say, $\{v(\Lambda)\}_{\Lambda \in \mathrm{UD}_{n, \lambda}(-2 m)}$, such that $v(\Lambda) L_{a}=\operatorname{res}_{\Lambda}(a) v(\Lambda)$, for each integer a with $1 \leq a \leq n$.

Proof. By definition, $V^{\lambda}$ is an irreducible representation of the semisimple $\mathbb{C}$ algebra $C_{n}(m):=\operatorname{End}_{S p_{2 m}(\mathbb{C})}\left(V^{\otimes n}\right)$ corresponding to $\lambda$. Note that there is a natural embedding $C_{n-1}(m) \hookrightarrow C_{n}(m)$. By results of Wenzl [18], we have that

$$
\pi_{n-1}: \quad V^{\lambda} \downarrow_{C_{n-1}(m)} \cong \bigoplus_{\mu} V^{\mu},
$$

where the subscript runs over all the partitions $\mu$ such that $\mu_{1} \leq m$ and the Young diagram $[\mu]$ is obtained from the Young diagram $[\lambda]$ by either adding a box or removing a box.

We use induction on $n$. By the induction hypothesis, such a canonical basis is already well-defined for each irreducible representation $V^{\mu}$ of $C_{n-1}(m)$ (or equivalently, of $B_{n-1}(-2 m)$ ). Using the isomorphism of (3.7), we get a basis of $V^{\lambda}$. Applying Corollary 3.3 , it is easy to see that this basis has the desired property. This completes the proof of the theorem.

LEMMA 3.8. Let $\Lambda, \Lambda^{\prime}$ be any two $(-2 m)$-permissible up-down tableaux of length $n$, and $a$ be an integer with $1 \leq a \leq n$ such that $\operatorname{res}_{\Lambda}(a)=\operatorname{res}_{\Lambda^{\prime}}(a)$.

(1) If $\Lambda(a-1)=\Lambda^{\prime}(a-1)$, then $\Lambda(a)=\Lambda^{\prime}(a)$.

(2) If $\Lambda(a)=\Lambda^{\prime}(a)$, then $\Lambda(a-1)=\Lambda^{\prime}(a-1)$.

Proof. Suppose that $\Lambda(a-1)=\Lambda^{\prime}(a-1)$. If $\Lambda(a), \Lambda^{\prime}(a) \supset \Lambda(a-1)$ or $\Lambda(a)$, $\Lambda^{\prime}(a) \subset \Lambda(a-1)$, then it is clear that $\operatorname{res}_{\Lambda}(a)=\operatorname{res}_{\Lambda^{\prime}}(a)$ implies that $\Lambda(a)=\Lambda^{\prime}(a)$. Now without loss of generality, we assume that $\Lambda(a) \supset \Lambda(a-1)$ and $\Lambda^{\prime}(a) \subset \Lambda(a-1)$. Let $(i, j)\left(\right.$ resp. $\left.\left(i^{\prime}, j^{\prime}\right)\right)$ be the box by which $\Lambda(a)$ (resp. $\left.\Lambda^{\prime}(a)\right)$ differs from $\Lambda(a-1)$. Then

$$
\operatorname{res}_{\Lambda}(a)-\operatorname{res}_{\Lambda^{\prime}}(a)=-2 m-1+j+j^{\prime}-i-i^{\prime} \leq-2 m-1+m+m-i-i^{\prime}<0 .
$$

So $\operatorname{res}_{\Lambda}(a) \neq \operatorname{res}_{\Lambda^{\prime}}(a)$, which is a contradiction. This proves (1). The proof of (2) is similar and left to the readers. 
COROLlARY 3.9. Let $\Lambda, \Lambda^{\prime}$ be any two $(-2 m)$-permissible up-down tableaux of length $n$. Suppose that for each integer a with $1 \leq a \leq n$, $\operatorname{res}_{\Lambda}(a)=\operatorname{res}_{\Lambda^{\prime}}(a)$. Then $\Lambda=\Lambda^{\prime}$.

The following corollary is similar to $[\mathbf{1 5}, 2.7]$. But note that in our case we have no restriction on $m$.

COROLlary 3.10. (1) The image in $C_{n}(m)$ of the Jucys-Murphy operators $L_{1}, \ldots$, $L_{n}$ generate a maximal commutative subalgebra of $C_{n}(m)$.

(2) The images in $C_{n}(m)$ of the elements $L_{1}^{i}+\cdots+L_{n}^{i}$ with $i=1,3, \ldots$ generate the center of $C_{n}(m)$.

Proof. For any integer $f$ with $0 \leq f \leq[n / 2]$ and any partition $\lambda$ of $n-2 f$ satisfying $\lambda_{1} \leq m$, we define $n_{\lambda}:=\# \mathrm{UD}_{n, \lambda}(-2 m)$. Due to Theorem 3.6 and Lemma 3.8, there is a natural isomorphism

$$
C_{n}(m) \stackrel{\sim}{\longrightarrow} \bigoplus_{f=0}^{[n / 2]} \bigoplus_{\substack{\lambda \vdash n-2 f \\ \lambda_{1} \leq m}} \operatorname{End}\left(V^{\lambda}\right) \cong \bigoplus_{f=0}^{[n / 2]} \bigoplus_{\substack{\lambda \vdash n-2 f \\ \lambda_{1} \leq m}} M_{n_{\lambda}}(\mathbb{C}),
$$

such that the subalgebra of $C_{n}(m)$ generated by $L_{1}, \ldots, L_{n}$ is mapped onto the subalgebra consists of all the diagonal matrices. This proves (1). (2) can be proved in a similar way as the proof of $[15,2.7]$. The only difference is that we require no restriction on $m$ in our case.

4. Identifying $V^{\lambda}$ with $\widetilde{D}^{f, \lambda}$. In this section, we shall identify those irreducible representations $V^{\lambda}$ found in $V^{\otimes n}$ with the irreducible representations constructed from a cellular basis of $B_{n}(-2 m)$.

By [7] (see also [5]), the Brauer's centralizer algebra $B_{n}(x)$ (for any parameter $x$ ) is always a cellular algebra. Throughout, we shall only be concerned with the case where $x=-2 m$. To describe its cellular structure, we need some combinatorial notations. For each integer $f$ with $0 \leq f \leq[n / 2]$, and each partition $\lambda \vdash n-2 f$, we denote by $\operatorname{Std}(\lambda)$ the set of all the standard $\lambda$-tableaux with entries in $\{2 f+1,2 f+2, \ldots, n\}$. For simplicity, we shall abbreviate the partition $(\underbrace{2, \ldots, 2})$ as $\left(2^{f}\right)$. We define $\underline{\lambda}:=\left(\left(2^{f}\right), \lambda\right)$, which is a bipartition of $n$. We also define $\mathfrak{t}^{f \text { copies }}:=\left(\mathfrak{t}^{\left(2^{f}\right)}, \mathfrak{t}^{\lambda}\right)$, where $\mathfrak{t}^{\left(2^{f}\right)}\left(\right.$ resp. $\left.\mathfrak{t}^{\lambda}\right)$ is the standard $\left(2^{f}\right)$-tableau (resp. standard $\lambda$-tableau) in which the numbers $\{1, \ldots, 2 f\}$ (resp. $\{2 f+1, \ldots, n\})$ are entered in increasing order from left to right along the rows.

DeFINITION 4.1. For each integer $f$ with $0 \leq f \leq[n / 2]$, and each partition $\lambda \vdash$ $n-2 f$, we define

$$
\mathcal{D}_{\underline{\lambda}}:=\left\{\begin{array}{l|l}
d \in \mathfrak{S}_{n} \mid \begin{array}{l}
\left(\mathfrak{t}^{(1)}, \mathfrak{t}^{(2)}\right)=\mathfrak{t}^{\lambda} d \text { is row standard and the first column of } \mathfrak{t}^{(1)} \text { is } \\
\text { an increasing sequence when read from top to bottom. }
\end{array}
\end{array}\right\} .
$$

Let $*$ be the anti-automorphism of $B_{n}(-2 m)$ which is defined on generators by $\sigma^{*}=\sigma^{-1}, e_{i}^{*}=e_{i}, \forall \sigma \in \mathfrak{S}_{n}, 1 \leq i<n$. By [5], the following set

$$
\left\{\begin{array}{l|l}
d_{1}^{*} e_{1} e_{3} \cdots e_{2 f-1} d(\mathfrak{s})^{*} x_{\lambda} d(\mathfrak{t}) d_{2} \mid \begin{array}{l}
0 \leq f \leq[n / 2], \lambda \vdash n-2 f, \mathfrak{s}, \mathfrak{t} \in \operatorname{Std}(\lambda), \\
d_{1}, d_{2} \in \mathcal{D}_{\underline{(n-2 f)}},
\end{array}
\end{array}\right\}
$$


where

$$
x_{\lambda}:=\sum_{\sigma \in \mathfrak{S}_{\left(2 f+1, \ldots, 2 f+\lambda_{1}\right)} \times \mathfrak{S}_{\left(2 f+\lambda_{1}+1, \ldots, 2 f+\lambda_{1}+\lambda_{2}\right)} \times \cdots} \sigma,
$$

and $d(\mathfrak{s}), d(\mathfrak{t}) \in \mathfrak{S}_{(2 f+1, \ldots, n)}$ with $\mathfrak{t}^{\lambda} d(\mathfrak{s})=\mathfrak{s}, \mathfrak{t}^{\lambda} d(\mathfrak{t})=\mathfrak{t}$, forms a cellular basis of $B_{n}(-2 m)$.

We define a partial order on the set $\{(f, \lambda)\}$, where $0 \leq f \leq[n / 2]$ and $\lambda \vdash n-2 f$, as follows.

Definition 4.2. Say that $(f, \lambda)>(g, \mu)$, where $f, g$ are both integers with $0 \leq$ $f, g \leq[n / 2]$, and $\lambda \vdash n-2 f, \mu \vdash n-2 g$, if

$$
f>g \text { or } f=g \text { and } \lambda \triangleright \mu
$$

where " $\triangleright$ " is the usual dominance order (see [12]) defined on the set of all the partitions of a given integer.

For simplicity, we shall write $M_{\mathfrak{s}, \mathfrak{t}, d_{1}, d_{2}}^{(f, \lambda)}$ instead of $d_{1}^{*} e_{1} e_{3} \cdots e_{2 f-1} d(\mathfrak{s})^{*} x_{\lambda} d(\mathfrak{t})^{*} d_{2}$.

DEFINITION 4.3. For each integer $f$ with $0 \leq f \leq[n / 2]$, and each partition $\lambda \vdash$ $n-2 f$, we define

$$
\begin{aligned}
N^{\geq(f, \lambda)}:=\mathbb{C}-\operatorname{Span}\left\{\begin{array}{l|l}
M_{\mathfrak{u}, \mathfrak{v}, d_{1}, d_{2}}^{(g, \mu)} & \begin{array}{l}
0 \leq g \leq[n / 2], \quad \mu \vdash n-2 g, \\
(g, \mu) \geq(f, \lambda), \mathfrak{u}, \mathfrak{v} \in \operatorname{Std}(\mu)
\end{array}
\end{array}\right\}, \\
N^{>(f, \lambda)}:=\mathbb{C}-\operatorname{Span}\left\{\begin{array}{ll}
M_{\mathfrak{u}, \mathfrak{v}, d_{1}, d_{2}}^{(g, \mu)} & \begin{array}{l}
0 \leq g \leq[n / 2], \quad \mu \vdash n-2 g, \\
(g, \mu)>(f, \lambda), \mathfrak{u}, \mathfrak{v} \in \operatorname{Std}(\mu)
\end{array}
\end{array}\right\}, \\
m_{\lambda}:=e_{1} e_{3} \cdots e_{2 f-1} x_{\lambda},
\end{aligned}
$$

and the Specht module $\widetilde{S}^{(f, \lambda)}$ for $B_{n}(-2 m)$ is defined to be the right $B_{n}(-2 m)$-submodule of $B_{n}(-2 m) / N^{>(f, \lambda)}$ generated by $m_{\lambda}+N^{>(f, \lambda)}$.

For each integer $f$ with $0 \leq f \leq[n / 2]$, we define

$$
B^{f}:=\text { two-sided ideal of } B_{n}(-2 m) \text { generated by } e_{1} e_{3} \cdots e_{2 f-1} \text {. }
$$

By the general theory of cellular algebra, there is a bilinear form naturally defined on $\widetilde{S}^{(f, \lambda)}$, say, $\langle$,$\rangle . We denote by \operatorname{rad}\langle$,$\rangle the radical of this form.$

We define $\widetilde{D}^{(f, \lambda)}:=\widetilde{S}^{(f, \lambda)} / \operatorname{rad}\langle$,$\rangle . Since we are working over the complex numbers$ field $\mathbb{C}$, it follows from [7, (4.17)] that $\widetilde{D}^{(f, \lambda)} \neq 0$ for each integer $f$ with $0 \leq f \leq[n / 2]$, and each partition $\lambda \vdash n-2 f$. Hence the set

$$
\left\{\widetilde{D}^{(f, \lambda)} \mid 0 \leq f \leq[n / 2], \lambda \vdash n-2 f\right\}
$$

forms a complete set of pairwise non-isomorphic absolutely irreducible $B_{n}(-2 m)$ modules.

THEOREM 4.4. For each integer $f$ with $0 \leq f \leq[n / 2]$, and each partition $\lambda \vdash n-2 f$ with $\lambda_{1} \leq m$, there is a nonzero $B_{n}(-2 m)$-module homomorphism from $\widetilde{S}^{(f, \lambda)}$ to $V^{\otimes n}$. In particular, $\widetilde{D}^{(f, \lambda)}$ is isomorphic to an irreducible $B_{n}(-2 m)$-submodule of $V^{\otimes n}$. 
Proof. By definition, the set

$$
\left\{d(\mathfrak{s})^{*} x_{\mu} d(\mathfrak{t}) \mid \mu \vdash n-2 f, \mathfrak{s}, \mathfrak{t} \in \operatorname{Std}(\mu)\right\},
$$

where

$$
x_{\mu}:=\sum_{\sigma \in \mathfrak{S}_{\left(2 f+1, \ldots, 2 f+\mu_{1}\right)} \times \mathfrak{S}_{\left(2 f+\mu_{1}+1, \ldots, 2 f+\mu_{1}+\mu_{2}\right)} \times \cdots} \sigma,
$$

forms a cellular basis of the symmetric group algebra $\mathbb{C S}_{(2 f+1, \ldots, n)}$. Let $S^{\lambda}$ be the Specht module (over $\mathfrak{S}_{(2 f+1, \ldots, n)}$ ) corresponding to $\lambda$ defined via the above cellular basis. Then $S^{\lambda}$ is generated (as right $\mathfrak{S}_{(2 f+1, \ldots, n)}$-module) by $x_{\lambda}+N^{>\lambda}$, where

$$
N^{>\lambda}:=\mathbb{C}-\operatorname{Span}\left\{d(\mathfrak{s})^{*} x_{\mu} d(\mathfrak{t}) \mid \mathfrak{s}, \mathfrak{t} \in \operatorname{Std}(\mu), \mu \triangleright \lambda\right\}
$$

Let $U$ be the subspace of $V$ generated by $v_{1}, \ldots, v_{m}$, then $U^{\otimes^{n-2 f}}$ naturally becomes a subspace of $V^{\otimes^{n-2 f}}$.

Let $\Lambda(m, n-2 f)$ be the set of compositions $\mu$ of $n-2 f$ such that $\ell(\mu) \leq m$. For each composition $\mu \in \Lambda(m, n-2 f)$, we define

$$
\begin{aligned}
y_{\mu} & :=\sum_{\sigma \in \mathfrak{S}_{\left(2 f+1, \ldots, 2 f+\mu_{1}\right)} \times \mathfrak{S}_{\left(2 f+\mu_{1}+1, \ldots, 2 f+\mu_{1}+\mu_{2}\right) \times \cdots}}(-1)^{\ell(\sigma)} \sigma, \\
v_{\mu} & :=\underbrace{v_{1} \otimes \cdots \otimes v_{1}}_{\mu_{1} \text { copies }} \otimes \cdots \otimes \underbrace{v_{m} \otimes \cdots \otimes v_{m}}_{\mu_{m} \text { copies }} \in U^{\otimes^{n-2 f}} .
\end{aligned}
$$

Let $\mathcal{D}_{\mu}$ be the set of distinguished right coset representatives of $\mathfrak{S}_{\left(2 f+1, \ldots, 2 f+\mu_{1}\right)} \times$ $\mathfrak{S}_{\left(2 f+\mu_{1}+1, \ldots, 2 f+\mu_{1}+\mu_{2}\right)} \times \cdots$ in $\mathfrak{S}_{(2 f+1, \ldots, n)}$. Then it is easy to see that the map which sends each $y_{\mu} d$ (where $d \in \mathcal{D}_{\mu}$ ) to $v_{\mu} d$ extends linearly to a right $\mathfrak{S}_{(2 f+1, \ldots, n)}$-module isomorphism $\phi: \bigoplus_{\mu \in \Lambda(m, n-2 f)} y_{\mu} \mathbb{C S}_{(2 f+1, \ldots, n)} \cong U^{\otimes^{n-2 f}}$.

Note that $\lambda^{\prime} \in \Lambda(m, n-2 f)$. Denote by $\mathfrak{t}^{\lambda^{\prime}}$ (resp. $\mathfrak{t}_{\lambda^{\prime}}$ ) the standard $\lambda^{\prime}$-tableau in which the numbers $2 f+1, \ldots, n$ appear in order along successive rows (resp. columns). Let $w_{\lambda^{\prime}}$ be the element in $\mathfrak{S}_{(2 f+1, \ldots, n)}$ such that $\mathfrak{t}^{\lambda^{\prime}} w_{\lambda^{\prime}}=\mathfrak{t}_{\lambda^{\prime}}$. Clearly, $w_{\lambda^{\prime}} \in \mathcal{D}_{\lambda^{\prime}}$. By $[\mathbf{1 3}$, (4.12)], $y_{\lambda^{\prime}} N^{>\lambda}=0$. Therefore the map which sends $x_{\lambda}+N^{>\lambda}$ to $v_{\lambda^{\prime}} w_{\lambda^{\prime}} x_{\lambda}$ extends naturally to a right $\mathfrak{S}_{(2 f+1, \ldots, n)}$-module homomorphism $\pi_{\lambda}: S^{\lambda} \rightarrow U^{\otimes^{n-2 f}} \hookrightarrow V^{\otimes^{n-2 f}}$. Moreover, as $v_{\lambda^{\prime}} w_{\lambda^{\prime}} x_{\lambda}=\phi\left(y_{\lambda^{\prime}} w_{\lambda^{\prime}} x_{\lambda}\right) \neq 0$, we see that $\pi_{\lambda}$ is nonzero.

We define

$$
v_{0}:=(\underbrace{\underbrace{v_{1} \otimes v_{1^{\prime}}} \otimes \cdots \otimes \underbrace{v_{1} \otimes v_{1^{\prime}}}}_{f \text { copies }}) \otimes v_{\lambda^{\prime}} w_{\lambda^{\prime}} \in V^{\otimes n} .
$$

We claim that the map $\pi$ which sends $e_{1} e_{3} \cdots e_{2 f-1} x_{\lambda}+N^{>(f, \lambda)}$ to

$$
v_{0} e_{1} e_{3} \cdots e_{2 f-1} x_{\lambda}=\left(v_{1} \otimes v_{1^{\prime}} \otimes \cdots \otimes v_{1} \otimes v_{1^{\prime}}\right) e_{1} e_{3} \cdots e_{2 f-1} \otimes v_{\lambda^{\prime}} w_{\lambda^{\prime}} x_{\lambda}
$$

naturally extends to a nonzero $B_{n}(-2 m)$-module homomorphism from $\widetilde{S}^{(f, \lambda)}$ to $V^{\otimes n}$.

It suffices to show that if $e_{1} e_{3} \cdots e_{2 f-1} x_{\lambda} h \in N^{>(f, \lambda)}$ for some $h \in B_{n}(-2 m)$, then $v_{0} e_{1} e_{3} \cdots e_{2 f-1} x_{\lambda} h=0$. 
In fact, if $e_{1} e_{3} \cdots e_{2 f-1} x_{\lambda} h \in N^{>(f, \lambda)}$ for some $h \in B_{n}(-2 m)$, then by the definition of multiplication in terms of concatenation of Brauer $n$-diagrams, it is easy to see that

$$
e_{1} e_{3} \cdots e_{2 f-1} x_{\lambda} h=e_{1} e_{3} \cdots e_{2 f-1}\left(\sum_{\substack{\mu \vdash n-2 f, \mu \triangleright \lambda \\ \mathfrak{s}, \mathfrak{t} \in \operatorname{Std}(\mu), d_{2} \in \mathcal{D}_{\underline{\mu}}}} d(\mathfrak{s})^{*} x_{\mu} d(\mathfrak{t}) d_{2}\right)+h_{2},
$$

where $h_{2} \in B^{f+1}$.

Therefore

$$
\begin{aligned}
v_{0} e_{1} e_{3} \cdots e_{2 f-1} x_{\lambda} h= & \sum_{\substack{\mu \vdash n-2 f, \mu \triangleright \lambda \\
\mathfrak{s}, \mathfrak{t} \in \operatorname{Std}(\mu), d_{2} \in \mathcal{D}_{\underline{\mu}}}}\left\{\left(\left(v_{1} \otimes v_{1^{\prime}} \otimes \cdots \otimes v_{1} \otimes v_{1^{\prime}}\right) e_{1} e_{3} \cdots e_{2 f-1}\right) \otimes\right. \\
& \left.\left(v_{\lambda^{\prime}} w_{\lambda^{\prime}} d(\mathfrak{s})^{*} x_{\mu} d(\mathfrak{t})\right)\right\} d_{2}+v_{0} h_{2} \\
= & 0,
\end{aligned}
$$

as required.

Now by the theory of cellular algebra, $\widetilde{D}^{(f, \lambda)}$ is the unique simple $B_{n}(-2 m)$-head of $\widetilde{S}^{(f, \lambda)}$. Hence $\widetilde{D}^{(f, \lambda)}$ is also the unique simple $B_{n}(-2 m)$-head of $\pi\left(\widetilde{S}^{(f, \lambda)}\right) \neq 0$. It follows that $\widetilde{D}^{(f, \lambda)}$ is isomorphic to an irreducible $B_{n}(-2 m)$-submodule of $V^{\otimes n}$. This completes the proof of the theorem.

DeFINITION 4.5. For each integer $f$ with $0 \leq f \leq[n / 2]$, and each partition $\lambda=$ $\left(\lambda_{1}, \lambda_{2}, \ldots\right)$ of $n-2 f$ with $\lambda_{1} \leq m$, we denote by $\Lambda_{f, \lambda}$ the following $(-2 m)$-permissible up-down tableau of shape $\lambda$ and length $n$ :

$$
\Lambda_{f, \lambda}:=(\underbrace{\emptyset,(1)}, \underbrace{\emptyset,(1)}_{\text {up tableau corresponding to } t^{\lambda}}, \ldots, \underbrace{\varnothing,(1)}, \underbrace{\emptyset,(1),(2), \ldots,\left(\lambda_{1}\right),\left(\lambda_{1}, 1\right), \ldots, \lambda}) .
$$

By [4, (3.12)], we have the following result.

THEOREM $4.6([4,(3.12)])$. For each integer $f$ with $0 \leq f \leq[n / 2]$, each partition $\lambda \vdash n-2 f$, and each integer $a$ with $1 \leq a \leq n$, we have that

$$
e_{1} e_{3} \cdots e_{2 f-1} x_{\lambda} L_{a} \equiv \operatorname{res}_{\Lambda_{f, \lambda}}(a) e_{1} e_{3} \cdots e_{2 f-1} x_{\lambda} \quad\left(\bmod N^{>(f, \lambda)}\right) .
$$

Now we can give the main result in this section.

THEOREM 4.7. For each integer $f$ with $0 \leq f \leq[n / 2]$, and each partition $\lambda \vdash n-2 f$ with $\lambda_{1} \leq m$, there is a $B_{n}(-2 m)$-module isomorphism from $\widetilde{D}^{(f, \lambda)}$ onto $V^{\lambda}$.

Proof. By Theorem 4.4, we know that $\widetilde{D}^{(f, \lambda)}$ is isomorphic (as $B_{n}(-2 m)$-module) to $V^{\mu}$ for some partition $\mu \vdash n-2 g$ satisfying $\mu_{1} \leq m$, where $g$ is an integer with $0 \leq g \leq[n / 2]$. It remains to show that $f=g$ and $\lambda=\mu$.

Let $\theta$ be the $B_{n}(-2 m)$-module isomorphism $\widetilde{D}^{(f, \lambda)} \cong V^{\mu}$. Let $\left[m_{\lambda}\right]$ be the image of $e_{1} e_{3} \cdots e_{2 f-1} x_{\lambda}+N^{>(f, \lambda)}$ in $\widetilde{D}^{(f, \lambda)}$. Suppose that

$$
\theta\left(\left[m_{\lambda}\right]\right)=\sum_{\Lambda \in \mathrm{UD}_{n, \mu}(-2 m)} a_{\Lambda} v(\Lambda)
$$

where $a_{\Lambda} \in \mathbb{C}$ for each $\Lambda \in \mathrm{UD}_{n, \mu}(-2 m)$. 
Let $\mathrm{UD}_{n}(-2 m)$ be the set of all the $(-2 m)$-permissible up-down tableaux of length $n$. We define

$$
\widetilde{F}:=\prod_{a=1}^{n} \prod_{\substack{\Sigma \in \operatorname{UD}_{n}(-2 m) \\ \operatorname{res}_{\Sigma}(a) \neq \operatorname{res}_{\Lambda_{f, \lambda}}(a)}} \frac{L_{a}-\operatorname{res}_{\Sigma}(a)}{\operatorname{res}_{\Lambda_{f, \lambda}}(a)-\operatorname{res}_{\Sigma}(a)} .
$$

By Theorem 4.6, $\left[m_{\lambda}\right] \widetilde{F}=\left[m_{\lambda}\right]$. By Corollary 3.9, it is easy to see that $v(\Lambda) \widetilde{F}=$ $\delta_{\Lambda, \Lambda_{f, \lambda}} v\left(\Lambda_{f, \lambda}\right)$. Applying $\widetilde{F}$ to both sides of (4.8), we get that $a_{\Lambda} \neq 0$ unless $\Lambda=\Lambda_{f, \lambda}$. In particular, we get that $f=g$ and $\lambda=\mu$. This completes the proof of the theorem.

5. The matrix elements. In this section, we shall, following the approach of [15], give an explicit description of the action of each generator of $B_{n}(-2 m)$ on the basis $\{v(\Lambda)\}_{\Lambda \in \mathrm{UD}_{n, \lambda}(-2 m)}$ (see Theorem 3.6) of the irreducible representations $V^{\lambda}$.

Let $f$ be an integer with $0 \leq f \leq[n / 2], \lambda$ be a partition of $n-2 f$ satisfying $\lambda_{1} \leq m$. For each integer $k$ with $1 \leq k<n$ and $\Lambda, \Lambda^{\prime} \in \mathrm{UD}_{n, \lambda}(-2 m)$, there are matrix elements $A_{k}\left(\Lambda, \Lambda^{\prime}\right), B_{k}\left(\Lambda, \Lambda^{\prime}\right) \in \mathbb{C}$, such that

$$
\left\{\begin{array}{l}
v(\Lambda) s_{k}=\sum_{\Lambda^{\prime} \in \mathrm{UD}_{n, \lambda}(-2 m)} A_{k}\left(\Lambda, \Lambda^{\prime}\right) v\left(\Lambda^{\prime}\right), \\
v(\Lambda) e_{k}=\sum_{\Lambda^{\prime} \in \mathrm{UD}_{n, \lambda}(-2 m)} B_{k}\left(\Lambda, \Lambda^{\prime}\right) v\left(\Lambda^{\prime}\right) .
\end{array}\right.
$$

Our purpose is to determine these $A_{k}\left(\Lambda, \Lambda^{\prime}\right), B_{k}\left(\Lambda, \Lambda^{\prime}\right)$. Let $\Lambda \in \operatorname{UD}_{n, \lambda}(-2 m)$, $k \in\{1, \ldots, n-1\}$ be fixed. Let $V^{\lambda}(k)$ be the subspace in $V^{\lambda}$ spanned by the vectors $v\left(\Lambda^{\prime}\right)$ such that $\Lambda^{\prime}(l)=\Lambda(l)$ for any $l \neq k$.

THEOREM 5.1. $A_{k}\left(\Lambda, \Lambda^{\prime}\right)=0$ (resp. $B_{k}\left(\Lambda, \Lambda^{\prime}\right)=0$ ) unless $v\left(\Lambda^{\prime}\right) \in V^{\lambda}(k)$. In particular, the subspace $V^{\lambda}(k)$ is stable under the actions of $s_{k}$ and $e_{k}$.

Proof. We define

$$
F_{\Lambda}:=\prod_{\substack{1 \leq a \leq n \\ a \neq k, k+1}} \prod_{\substack{\Sigma \in \operatorname{UD}_{n, \lambda}(-2 m) \\ \operatorname{res}_{\Sigma}(a) \neq \operatorname{res}_{\Lambda}(a)}} \frac{L_{a}-\operatorname{res}_{\Sigma}(a)}{\operatorname{res}_{\Lambda}(a)-\operatorname{res}_{\Sigma}(a)} .
$$

For any $v\left(\Lambda^{\prime}\right) \in V^{\lambda}(k)$, we have that

$$
v\left(\Lambda^{\prime}\right) s_{k}=\sum_{\Lambda^{\prime \prime} \in \mathrm{UD}_{n, \lambda}(-2 m)} A_{k}\left(\Lambda^{\prime}, \Lambda^{\prime \prime}\right) v\left(\Lambda^{\prime \prime}\right) .
$$

By definition and $[\mathbf{1 5},(2.3)], s_{k} F_{\Lambda}=F_{\Lambda} s_{k}$. Moreover, by Lemma 3.8 and the fact that $\Lambda(0)=\Lambda^{\prime \prime}(0)=\emptyset, \Lambda(n)=\Lambda^{\prime \prime}(n)=\lambda$,

$$
v\left(\Lambda^{\prime \prime}\right) F_{\Lambda}= \begin{cases}v\left(\Lambda^{\prime \prime}\right) & \text { if } v\left(\Lambda^{\prime \prime}\right) \in V^{\lambda}(k), \\ 0 & \text { otherwise. }\end{cases}
$$

Applying $F_{\Lambda}$ to (5.3), one can easily see that $A_{k}\left(\Lambda, \Lambda^{\prime}\right)=0$ unless $v\left(\Lambda^{\prime}\right) \in V^{\lambda}(k)$. The same argument shows that $V^{\lambda}(k)$ is stable under the action of $s_{k}$. In a similar way, one 
can show that $B_{k}\left(\Lambda, \Lambda^{\prime}\right)=0$ unless $v\left(\Lambda^{\prime}\right) \in V^{\lambda}(k)$, and $V^{\lambda}(k)$ is also stable under the action of $e_{k}$.

Lemma 5.4. For any integers $k, l \in\{1, \ldots, n\}$, let $(i, j)$ (resp. $\left.\left(i^{\prime}, j^{\prime}\right)\right)$ be the box by which $\Lambda(k)($ resp. $\Lambda(l))$ differs from $\Lambda(k-1)$ (resp. from $\Lambda(l-1))$.

(1) If $\operatorname{res}_{\Lambda}(k)+\operatorname{res}_{\Lambda}(l)=0$, then $j-i=j^{\prime}-i^{\prime}$, and either $(i, j)$ is a removable box of $[\Lambda(k-1)]$ and $\left(i^{\prime}, j^{\prime}\right)$ is an addable box of $[\Lambda(l-1)]$, or $(i, j)$ is an addable box of $[\Lambda(k-1)]$ and $\left(i^{\prime}, j^{\prime}\right)$ is a removable box of $[\Lambda(l-1)]$.

(2) If $\operatorname{res}_{\Lambda}(k)-\operatorname{res}_{\Lambda}(l)=0$, then $j-i=j^{\prime}-i^{\prime}$, and either $(i, j)$ is a removable box of $[\Lambda(k-1)]$ and $\left(i^{\prime}, j^{\prime}\right)$ is a removable box of $[\Lambda(l-1)]$, or $(i, j)$ is an addable box of $[\Lambda(k-1)]$ and $\left(i^{\prime}, j^{\prime}\right)$ is an addable box of $[\Lambda(l-1)]$.

Proof. This is easy to check by using the fact that $j+j^{\prime} \leq 2 \mathrm{~m}$.

COROLlary 5.5. For any integers $k \in\{1, \ldots, n-1\}$, we have that

(1) if $\operatorname{res}_{\Lambda}(k)+\operatorname{res}_{\Lambda}(k+1)=0$, then $\Lambda(k-1)=\Lambda(k+1)$,

(2) $\operatorname{res}_{\Lambda}(k) \neq \operatorname{res}_{\Lambda}(k+1)$.

THEOREM 5.6. Suppose that $\Lambda(k-1) \neq \Lambda(k+1)$, then $B_{k}\left(\Lambda, \Lambda^{\prime}\right)=0$ for any $\Lambda^{\prime} \in$ $\mathrm{UD}_{n, \lambda}(-2 m)$.

Proof. Suppose that $\Lambda(k-1) \neq \Lambda(k+1)$. By [15, (2.5)], $\left(L_{k}+L_{k+1}\right) e_{k}=0$. By 5.5.1, $\operatorname{res}_{\Lambda}(k)+\operatorname{res}_{\Lambda}(k+1) \neq 0$. Then it is easy to see that $v(\Lambda) e_{k}=0$, as required.

THEOREM 5.7. If $\Lambda(k-1) \neq \Lambda(k+1)$, then

(1) $A_{k}(\Lambda, \Lambda)=\left(\operatorname{res}_{\Lambda}(k+1)-\operatorname{res}_{\Lambda}(k)\right)^{-1}$;

(2) if $v\left(\Lambda^{\prime}\right) \in V^{\lambda}(k)$ with $\Lambda \neq \Lambda^{\prime}$, then

$$
A_{k}\left(\Lambda, \Lambda^{\prime}\right) A_{k}\left(\Lambda^{\prime}, \Lambda\right)=1-\left(\operatorname{res}_{\Lambda}(k+1)-\operatorname{res}_{\Lambda}(k)\right)^{-2} .
$$

Proof. This can be proved directly in the same way as [15, (3.2), (3.3)] by using the relations $s_{k} L_{k+1}-L_{k} s_{k}=1-e_{k}, s_{k}^{2}=1$ and the fact that the space $V^{\lambda}(k)$ has dimension at most two. (3.6)].

The following two lemmas can also be proved in exactly the same way as $[\mathbf{1 5},(3.5)$,

LEMMA 5.8. If $\Lambda(k-1)=\Lambda(k+1) ;$ then

$$
B_{k}(\Lambda, \Lambda)=\frac{\operatorname{dim} U^{\Lambda(k)}}{\operatorname{dim} U^{\Lambda(k+1)}} .
$$

In particular, $B_{k}(\Lambda, \Lambda)$ is explicitly known (as $\operatorname{dim} U^{\Lambda(k)}$ and $\operatorname{dim} U^{\Lambda(k+1)}$ are already known by [6]).

Lemma 5.9. Suppose that $\Lambda(k-1)=\Lambda(k+1)$, then the image of the action of $e_{k}$ in the subspace $V^{\lambda}(k)$ is one-dimensional.

Corollary 5.10. Suppose that $\Lambda(k-1)=\Lambda(k+1)$ and $v\left(\Lambda^{\prime}\right) \in V^{\lambda}(k)$. Then $B_{k}\left(\Lambda, \Lambda^{\prime}\right) B_{k}\left(\Lambda^{\prime}, \Lambda\right)=B_{k}(\Lambda, \Lambda) B_{k}\left(\Lambda^{\prime}, \Lambda^{\prime}\right)$.

Proof. We have that

$$
\begin{aligned}
v(\Lambda) e_{k} & =B_{k}(\Lambda, \Lambda) v(\Lambda)+B_{k}\left(\Lambda, \Lambda^{\prime}\right) v\left(\Lambda^{\prime}\right)+\cdots \\
v\left(\Lambda^{\prime}\right) e_{k} & =B_{k}\left(\Lambda^{\prime}, \Lambda^{\prime}\right) v\left(\Lambda^{\prime}\right)+B_{k}\left(\Lambda^{\prime}, \Lambda\right) v(\Lambda)+\cdots
\end{aligned}
$$


By Lemma 5.9, $v(\Lambda) e_{k}$ is a scalar multiple of $v\left(\Lambda^{\prime}\right) e_{k}$. It follows that

$$
B_{k}\left(\Lambda, \Lambda^{\prime}\right) B_{k}\left(\Lambda^{\prime}, \Lambda\right)=B_{k}(\Lambda, \Lambda) B_{k}\left(\Lambda^{\prime}, \Lambda^{\prime}\right)
$$

THEOREM 5.11. Suppose that $\Lambda(k-1)=\Lambda(k+1)$ and $v\left(\Lambda^{\prime}\right) \in V^{\lambda}(k)$. Then $\operatorname{res}_{\Lambda}(k)+\operatorname{res}_{\Lambda^{\prime}}(k) \neq 0$, and

$$
A_{k}\left(\Lambda, \Lambda^{\prime}\right)=\left(B_{k}\left(\Lambda, \Lambda^{\prime}\right)-\delta_{\Lambda, \Lambda^{\prime}}\right)\left(\operatorname{res}_{\Lambda}(k)+\operatorname{res}_{\Lambda^{\prime}}(k)\right)^{-1}
$$

Proof. Let $(i, j)$ (resp. $\left.\left(i^{\prime}, j^{\prime}\right)\right)$ be the box by which $\Lambda(k)$ (resp. $\left.\Lambda^{\prime}(k)\right)$ differs from $\Lambda(k-1)$ (resp. from $\left.\Lambda^{\prime}(k-1)\right)$. Suppose that $\operatorname{res}_{\Lambda}(k)+\operatorname{res}_{\Lambda^{\prime}}(k)=0$. If either $(i, j)$ is a removable box of $[\Lambda(k-1)]$ and $\left(i^{\prime}, j^{\prime}\right)$ is a removable box of $\left[\Lambda^{\prime}(k-1)\right]$, or $(i, j)$ is an addable box of $[\Lambda(k-1)]$ and $\left(i^{\prime}, j^{\prime}\right)$ is an addable box of $\left[\Lambda^{\prime}(k-1)\right]$, then one has that

$$
0=\frac{-2 m-1}{2}+j-i+\frac{-2 m-1}{2}+j^{\prime}-i^{\prime}=-2 m-1+j+j^{\prime}-i-i^{\prime},
$$

which is impossible as $j+j^{\prime} \leq 2 m$. If either $(i, j)$ is a removable box of $[\Lambda(k-1)]$ and $\left(i^{\prime}, j^{\prime}\right)$ is an addable box of $\left[\Lambda^{\prime}(k-1)\right]$, or $(i, j)$ is a removable box of $[\Lambda(k-1)]$ and $\left(i^{\prime}, j^{\prime}\right)$ is an addable box of $\left[\Lambda^{\prime}(k-1)\right]$, then one has that

$$
0=\frac{-2 m-1}{2}+j-i-\frac{-2 m-1}{2}-j^{\prime}+i^{\prime}=(j-i)-\left(j^{\prime}-i^{\prime}\right) .
$$

Since $\Lambda(k-1)=\Lambda^{\prime}(k-1)$, it follows that $i=i^{\prime}$ and $j=j^{\prime}$, which is impossible. This proves that $\operatorname{res}_{\Lambda}(k)+\operatorname{res}_{\Lambda^{\prime}}(k) \neq 0$. The remaining part of this theorem can be proved directly in the same way as $[\mathbf{1 5},(3.11)]$ by using the relation $s_{k} L_{k}-L_{k+1} s_{k}=e_{k}-1$.

Note that each vector of our basis $\{v(\Lambda)\}_{\Lambda \in \mathrm{UD}_{n, \lambda}(-2 m)}$ is defined up to a scalar multiplier.

THEOREM 5.12. The basis $\{v(\Lambda)\}_{\Lambda \in \mathrm{UD}_{n, \lambda}(-2 m)}$ can be chosen so that for any $v\left(\Lambda^{\prime}\right) \in$ $V^{\lambda}(k)$ with $\Lambda \neq \Lambda^{\prime}$, (1) and (2) hold.

(1) If $\Lambda(k-1) \neq \Lambda(k+1)$, then $A_{k}\left(\Lambda, \Lambda^{\prime}\right)=A_{k}\left(\Lambda^{\prime}, \Lambda\right)>0$.

(2) If $\Lambda(k-1)=\Lambda(k+1)$, then $B_{k}\left(\Lambda, \Lambda^{\prime}\right)=B_{k}\left(\Lambda^{\prime}, \Lambda\right)>0$.

Proof. Let $\langle$,$\rangle be the non-degenerate symmetric bilinear form on V$ which is defined by $\left\langle v_{i}, v_{j}\right\rangle=\delta_{i, j}$ for any $i, j \in\left\{1, \ldots, m, m^{\prime}, \ldots, 1^{\prime}\right\}$. Taking products we get a non-degenerate symmetric bilinear form on $V^{\otimes n}$. We denote it by $\langle,\rangle_{n}$. It is easy to verify that the action of each generators of $B_{n}(-2 m)$ on $V^{\otimes n}$ is self-adjoint with respect to $\langle,\rangle_{n}$. Therefore, we get (by restriction) a non-degenerate symmetric bilinear form on the irreducible module $V^{\lambda}$. By construction, the isomorphism $\pi_{n-1}$ in (3.7) can be chosen such that

$$
\langle x, y\rangle_{n}=\left\langle\pi_{n-1}(x), \pi_{n-1}(y)\right\rangle_{n-1} .
$$

Note that our basis $\{v(\Lambda)\}_{\Lambda \in \mathrm{UD}_{n, \lambda}(-2 m)}$ was inductively defined via the isomorphism $\pi_{n-1}$. It follows that the basis $\{v(\Lambda)\}_{\Lambda \in \mathrm{UD}_{n, \lambda}(-2 m)}$ can be chosen such that $\left\langle v(\Lambda), v\left(\Lambda^{\prime}\right)\right\rangle_{n}=\delta_{\Lambda, \Lambda^{\prime}}$ for any $\Lambda, \Lambda^{\prime} \in \operatorname{UD}_{n, \lambda}(-2 m)$. In other words, the map which sends $v(\Lambda)$ to $v(\Lambda)^{*}$ for each $\Lambda^{\prime} \in \mathrm{UD}_{n, \lambda}(-2 m)$ defines a $B_{n}(-2 m)$-module isomorphism $V^{\lambda} \cong\left(V^{\lambda}\right)^{*}$. From this it follows that $A_{k}\left(\Lambda, \Lambda^{\prime}\right)=A_{k}\left(\Lambda^{\prime}, \Lambda\right)$ and $B_{k}\left(\Lambda, \Lambda^{\prime}\right)=B_{k}\left(\Lambda^{\prime}, \Lambda\right)$ for any $\Lambda, \Lambda^{\prime} \in \operatorname{UD}_{n, \lambda}(-2 m)$. The remaining part of this theorem can be proved in exactly the same way as the proof of $[\mathbf{1 5},(3.12)]$. 
Now Theorem 5.1, Theorem 5.6, Theorem 5.7, Lemma 5.8, Corollary 5.10, Theorem 5.11 and Theorem 5.12 completely determine all the matrix elements $B_{k}\left(\Lambda, \Lambda^{\prime}\right)$ and $A_{k}\left(\Lambda, \Lambda^{\prime}\right)$.

\section{REFERENCES}

1. R. Brauer, On algebras which are connected with semisimple continuous groups, Ann. of Math. (2) 38 (1937), 857-872.

2. W. P. Brown, An algebra related to the orthogonal group, Michigan Math. J. 3 (19551956), 1-22.

3. W. P. Brown, The semisimplicity of $\omega_{f}^{n}$, Ann. of Math. (2) 63 (1956), 324-335.

4. R. Dipper and J. Hu, Brauer's centralizer algebra and combinatorics of up-down tableaux, preprint.

5. J. Enyang, Bases of certain algebras associated with quantum groups, $\mathrm{Ph} . \mathrm{D}$. thesis, (University of Illinois at Chicago, 2002).

6. N. El Samra and R. C. King, Dimension of irreducible representations of the classical groups, J. Phys. A 12 (1979), 2317-2328.

7. J. J. Graham and G. I. Lehrer, Cellular algebras, Invent. Math. 123 (1996), 1-34.

8. R. Goodman and N. R. Wallach, Representations and invariants of classical groups, (Cambridge University Press, 1998).

9. P. Hanlon and D. B. Wales, On the decomposition of Brauer's centralizer algebras, J. Algebra 121 (1989), 409-445.

10. P. Hanlon and D. B. Wales, Eigenvalues connected with Brauer's centralizer algebras, J. Algebra 121 (1989), 446-475.

11. R. Leduc and A. Ram, A Ribbon Hopf algebra approach to the irreducible representations of centralizer algebras: the Brauer, Birman-Wenzl, and type $A$ Iwahori-Hecke algebras, Adv. Math. 125 (1997), 1-94.

12. A. Mathas, Iwahori-Hecke algebras and Schur algebras of the symmetric group, University Lecture Series, 15 (American Mathematical Society, Providence, RI, 1999). $97-121$.

13. E. Murphy, The representations of Hecke algebras of type $A_{n}, J$. Algebra 173 (1995),

14. J. Murakami, The Kauffman polynomial of links and representation theory, Osaka J. Math. (4) 26 (1987), 745-758.

15. M. Nazarov, Young's orthogonal form for Brauer's centralizer algebras, J. Algebra 182 (1996), 664-693. 200.

16. A. Ram, Characters of Brauer's centralizer algebras, Pacific. J. Math. 169 (1995), 173

17. H. Weyl, The classical groups, their invariants and representations (Princeton University Press, 1946).

18. H. Wenzl, On the structure of Brauer's centralizer algebras, Ann. of Math. (2) 128 (1988), 173-193. 